\title{
CURTISS ON COMPLEX VARIABLES
}

Analytic Functions of a Complex Variable. By D. R. Curtiss. Chicago, The Open Court Publishing Co., 1926. ix+173 pp. Price $\$ 2.00$.

The present volume is the second one in the series of Carus Monographs, the first being Professor Bliss's Calculus of Variations. The reader is assumed to have a preparation equivalent to elementary differential and integral calculus. However, without such preparation one may from the present volume, "obtain some idea of the scope and purpose of the theory of functions."

The purpose of the book seems to be admirably in line with that of Mrs. Mary Hegeler Carus, whose generosity has made these monographs possible. This purpose is appropriately described to be the "diffusion of mathematical and formal thought as contributory to exact knowledge and clear thinking not only for mathematicians and teachers of mathematics but also for other scientists and the public at large."

The author says that inasmuch as the theory of functions of a complex variable has been developed by thousands of workers through the last hundred years, it would be impossible to give an account of that theory in all of its branches within the limits of this book. He attempts to present "fundamental principles with sufficient details of proof and discussion to avoid the style of a mere summary ....."

Three practically equivalent points of view may be adopted in the treatment of the theory of analytic functions of a complex variable. One of these, that of Meray-Weierstrass, bases its developments on the properties of power series. Another is the Cauchy-Riemann point of view in which the functions are required to possess a differential quotient, that is, the definition is here made by a descriptive property rather than by a constructive process. The development may also be made from considerations of the curvilinear integral - the Cauchy-Morera point of view. The volume under review gives a rather interesting account primarily of the Cauchy-Riemann point of view with attention directed occasionally to other methodologies.

A brief survey of the contents of the book is as follows:

The first chapter gives an historical account of the development of the idea of analytic function and calls attention to the usefulness of the theory.

Chapter two deais with complex numbers. In its historical account, in its references to a more general number system, and in its general treatment, it is much more than an elementary exposition. The reviewer would like to have seen an even fuller account of the ideas embodied here, having in mind the fact that in many cases students and the general reader come up to a study of the theory of functions without ever having heard of DeMoivre's theorem and at the same time with very little working equip- 
ment in complex numbers. In this particular case it seems wiser to include this elementary material than to omit it.

Chapter three considers real functions. It treats the usual elementary topics in this field including limits, continuity, derivatives and integrals, functions of two variables, double integrals, curvilinear integrals, Green's lemma, integrals independent of the path, and implicit functions.

Chapter four introduces the Cauchy idea of analytic function as one having a derivative. It then discusses necessary and sufficient conditions for the existence of a derivative. The chapter ends with the definition of the exponential, the trigonometric and the logarithmic functions for complex values.

Chapter five, entitled "Applications in Geometry and Physics," gives the usual treatment of conformal representation and shows how the Laplace equation enters in the theory of fluid motion. It also indicates the connection of the mathematical theory with the flow of heat and logarithmic potential.

Chapter six defines complex integrals, deduces Cauchy's integral theorem and formula with the customary corollaries, and ends by deriving Taylor's theorem with remainder.

Chapter seven takes up infinite series, both real and complex. The reviewer would like to have seen here a fuller discussion of power series together with a clearer presentation of the relation between the Cauchy and the Weierstrass points of view.

Chapter eight deals with singularities of single-valued analytic functions, proving some important theorems concerning removable and essential singularities and the nature of the infinity at a pole. It closes with a discussion of definitions of functions in terms of their singularities.

Chapter nine is entitled "Analytic Continuation. Many-Valued Analytic Functions." Here, among other things, we find a discussion of analytic continuation in general and by the method of overlapping circles, monogenic analytic functions, the permanence of functional equations, branches and branch points, the monodromic group, and Riemann surfaces.

At the end of each chapter full and explicit page references to several standard treatises in English and one in German are given for additional reading on the various topics under discussion.

Such typographical errors, few in number, as do occur would perhaps be evident to the reader. For example, the first two subscripts in equation 10, p. 127 should be $-2,-1$.

The book is attractive in form, binding, and printing, and is an admirable introduction to the subject. Its mastery would most certainly induce the pleasure of further conquests in this beautiful theory.

S. E. RASOR 